\title{
On the origin of the ultra-high energy cosmic ray anisotropy
}

\author{
Noémie Globus \\ New York University/Flatiron Institute \\ E-mail: globus@nyu .edu \\ Chen Ding* \\ New York University \\ E-mail: cd2209@nyu.edu

\section{Glennys Farrar} \\ New York University \\ E-mail: glennys.farrarenyu.edu
}

We show that the observed anisotropy of extragalactic ultra-high energy cosmic rays (UHECRs above $8 \mathrm{EeV}$ ) is well-described by treating sources as a continuous distribution following the local large-scale structure of matter (LSS). We assume homogeneous, rigidity-dependent diffusion in the extragalactic magnetic field (EGMF), taking its strength and coherence length as free parameters. We use high-resolution particle tracking $\left(\sim 1.8 \times 10^{9}\right.$ trajectories $)$ to treat the impact of the Galactic magnetic field (GMF), for rigidities (E/Z) from $10^{17.4}-10^{20} \mathrm{EV}$. We adopt the Jansson and Farrar (2012) model for the GMF, and consider values of the coherence length of the random component from 30-100 pc. We describe the composition and spectrum of UHECRs using both direct fits from Auger (2017) and the physically-based Muzio, Unger, Farrar (2019) model, and assess the impact of UHECR spectrum and composition uncertainties on predicted arrival direction anisotropies. Our model accounts for the magnitude and direction of the dipole anisotropy in the $>8 \mathrm{EeV}$ bin, as well as its evolution (in both magnitude and direction) in the 8-16, 16-32 and $>32 \mathrm{EeV}$ bins (although the dipole is not significant above $32 \mathrm{EeV}$ due to the lack of statistics). The quadrupole is also in agreement with data. Our best fit parameters are $B_{\mathrm{EGMF}} \sim 0.6 \mathrm{nG}, \lambda_{\mathrm{EGMF}} \sim 0.2 \mathrm{Mpc}$ and $\lambda_{\mathrm{GMF}} \sim 75 \mathrm{pc}$. We show that this result is robust against different dipole reconstruction methods (Rayleigh/K-inverse) and different hadronic interaction models (EPOS-LHC/Sybill 2.3). We note that in an alternative scenario where the dipole would be due to a few local sources, the effect of cosmic variance would dominate over the magnetic deflection and horizon effects, and the direction and magnitude of the anisotropy could not be predicted. We also note that a pure proton composition would not explain simultaneously the strength and direction of the dipole in this model.

36th International Cosmic Ray Conference -ICRC2019-

July 24th - August 1st, 2019

Madison, WI, U.S.A.

${ }^{*}$ Speaker. 


\section{Introduction}

Recently, the Pierre Auger collaboration (hereafter Auger) has reported a large scale anisotropy above $8 \mathrm{EeV}[1,2]$. This discovery has been a breakthrough, because it is the first $5 \sigma$ anisotropy in the UHECR sky. The amplitude of the dipole anisotropy is $\approx 6 \%$ at $8-16 \mathrm{EeV}$ and increases with energy; the direction is rather well-constrained and initially seemed puzzling because it aligns neither with the CMB dipole nor the dipole in the flux of optical light from galaxies.

The amplitude and direction of UHECR anisotropies reflects the distribution of UHECR sources, modified by the intervening EGMF and GMF. If the dipole is due to a single nearby source (a few Mpc away, like Cen A) the diffusion in the EGMF would have to be sufficient to produce a dipole, requiring $B_{\mathrm{EGMF}} \sim \mathscr{O}(100) \mathrm{nG}[3,4]$. A second scenario, invoking a large number of sources following the LSS, requires $B_{\mathrm{EGMF}} \sim \mathscr{O}(1) \mathrm{nG}[5,6]$. In this scenario, the direction and amplitude of the dipole are modified by the EGMF due to the magnetic horizon effect [7]. Extending this study, Globus et al.[6] showed that the direction and magnitude of the dipole $>8 \mathrm{EeV}$ is qualitatively improved by the addition of GMF deflections, using the Jansson-Farrar 2012 (JF12) [8] Galactic magnetic field model ${ }^{1}$. However the study focused on a single energy bin $(>8 \mathrm{EeV})$ and considered exemplary choices of pure composition ( $\mathrm{H}, \mathrm{He}$ and $\mathrm{N})$. The latest Auger release [2] of the dipole amplitude and direction in four energy bins (4-8, 8-16, 16-32 and $>32 \mathrm{EeV})$ and the quadrupole amplitude in two energy bins ( $4-8$ and $>8 \mathrm{EeV})$, is a call for further investigations.

The main effect of the EGMF is to limit the size of the UHECR observable Universe. If the diffusion time $d_{\text {diff }} / c$ is larger than the Hubble time or the energy loss time $\sim d_{\mathrm{GZK}} / c$, the cosmic rays cannot reach us. The size of the cosmic-ray observable universe is referred to as 'cosmic-ray horizon' given by: $H(E)=\min \left(\sqrt{d_{\mathrm{diff}} d_{\mathrm{GZK}}}, d_{\mathrm{GZK}}\right)$ [6]. The diffusion of cosmic-rays in the magnetic field depends just on the rigidity of the nuclei, while the GZK distance depends on cosmic-ray mass and energy $[3,9]$. In the following we assume a purely homogeneous and isotropic turbulent EGMF with a Kolmogorov spectrum; our EGMF diffusion depends therefore only on the RMS field strength and coherence length.

The Galactic magnetic field has an ordered component and a turbulent component. The ordered component includes a large scale field that threads the Galactic disc and extends through the halo [8]. This induces shifts in the direction of the cosmic-ray entering the Galaxy and source magnification or de-magnification, depending on the source direction [10]. The GMF ordered component has mainly an effect on the UHECR dipole direction, while the GMF turbulent component mostly affects the dipole amplitude.

To quantify these effects, we perform here a full parametric analysis. Our different sets of model parameters are listed below:

- Different composition models (EPOS-LHC and Sibyll 2.3c, allowing shift in $<X_{\max }>$ ) using the [12] (hereafter MUF19) framework, as well as direct composition fits of Auger (EPOS-LHC and Sibyll 2.3) [13];

- Different diffusion coefficients in the extragalactic medium, assuming a purely turbulent EGMF with $0.1 \leq B_{\mathrm{EGMF}} \leq 30 \mathrm{nG}$ and coherence length $0.08 \leq \lambda_{\mathrm{EGMF}} \leq 0.5 \mathrm{Mpc}$;

- JF12 model for the GMF with coherence length of random component $30 \leq \lambda_{\mathrm{GMF}} \leq 100 \mathrm{pc}$.

\footnotetext{
${ }^{1}$ More precisely, the JF12 coherent field model and rescaled JF12 random field with $\lambda_{\mathrm{GMF}}=200 \mathrm{pc}$ (see[6] for more details).
} 


\section{Methods}

\subsection{Spectrum and composition}

We use i) the composition fit from depth of maximum of air-shower profiles by Auger [13] and ii) a physically motivated model which accounts for the observed energy spectrum and composition (MUF19) [12]. Besides providing a very general framework for describing cosmic ray sources, the MUF19 modeling allows precise fitting to the spectrum and composition data. We test the effect of different hadronic interaction models (EPOS-LHC and Sybill 2.3). We use the same energy binning as Auger. The rigidity distribution of all cosmic rays $>8 \mathrm{EeV}$, using the energy spectrum and composition from Auger for Sibyll and EPOS, is shown in the left panel of figure 1. The right panel shows the UHECR horizons for the different elements as a function of rigidity in an EGMF of $B_{\mathrm{EGMF}} \sim 0.6 \mathrm{nG}, \lambda_{\mathrm{EGMF}} \sim 0.2 \mathrm{Mpc}$. For rigidities between 2 and $4 \mathrm{EeV}$ the horizon is $\sim 300$ Mpc.
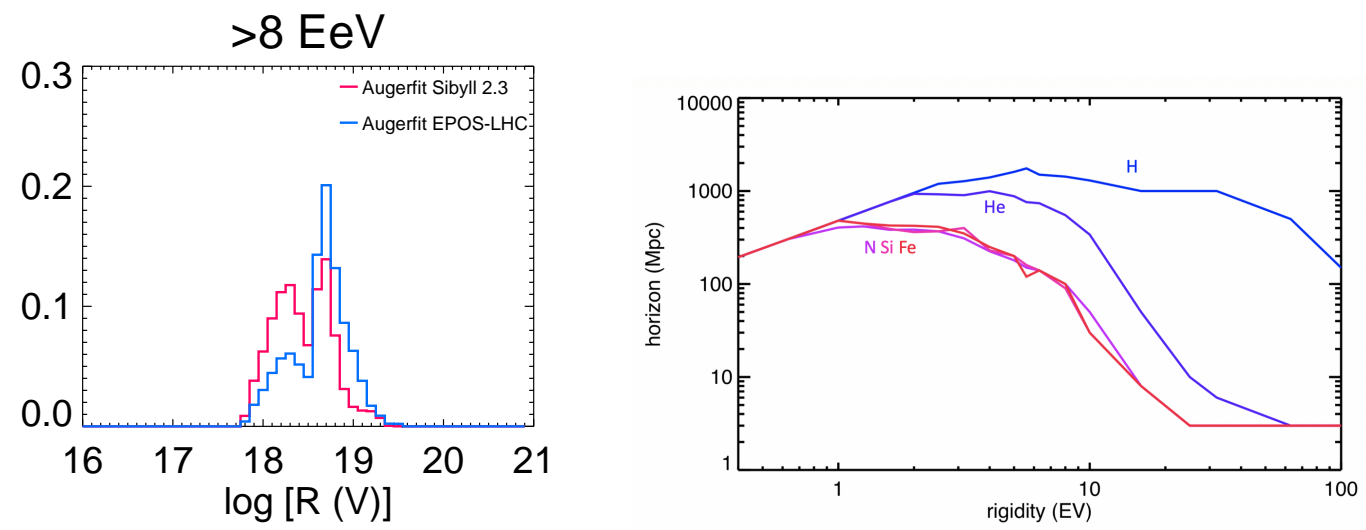

Figure 1: Left panel: rigidity spectrum for the $>8 \mathrm{EeV}$ energy bin based on Auger composition extraction using Sibyll (red) and EPOS (blue). Right panel: UHECR horizon as a function of rigidity, for different elements, for $B_{\mathrm{EGMF}} \sim 0.6$ $\mathrm{nG}, \lambda_{\mathrm{EGMF}} \sim 0.2 \mathrm{Mpc}$ (our best model parameters).

\subsection{Illumination map calculation procedure}

The illumination map (IM), defined as the sky map of flux illuminating the surface of the Milky Way, is an important intermediate concept in understanding the anisotropy. We use the reconstructed large-scale structure matter density field derived from the CosmicFlows-2 catalog of peculiar velocities [14]. The uncertainty in the reconstructed density field leads to an uncertainty in the dipole amplitude, which is argued in [6] to be less than about a percent; for further details see [6]. In calculation of the IM, we include diffusion in the EGMF and energy loss processes (explained in detail in [6]). To obtain the illumination map of a particular energy bin, we add up the IMs of different element bins and rigidity bins with weights given by the rigidity spectrum.

\subsection{Arrival map calculation procedure}

The arrival map (AM), defined as the sky map of CR flux at Earth, is what observatories measure and what our models predict. To account for the Galactic propagation, we used the JF12 Galactic magnetic field model [8]. Over 1.8 billion trajectories have been simulated [10] with two 
choices of coherence length of the Kolmogorov random field, $30 \mathrm{pc}$ and $100 \mathrm{pc}$. We simulated additional trajectories beyond [10] to span the full range of rigidity $(17.4 \leq \log R \leq 20.0)$ in 24 bins $^{2}$. At $\log R=17.4$, no small-angular-scale anisotropies appear in the illumination maps, and the GMF effectively isotropizes cosmic rays. Therefore we assume isotropic arrival maps for $\log R<$ 17.35. For this analysis, we also assume isotropic arrival for heavy-composition cosmic rays of Galactic origin in MUF19. In order to have more choices of GMF coherence lengths, we mix the trajectories of 30pc and 100pc with different weights as a reasonable interpolation. With the above GMF deflection data, we can predict the arrival map by adding up the AMs of different element bins and rigidity bins with weights given by the rigidity spectrum. Figure 2 shows the IM and AM for different energy bins, for the overall best-fit parameters.
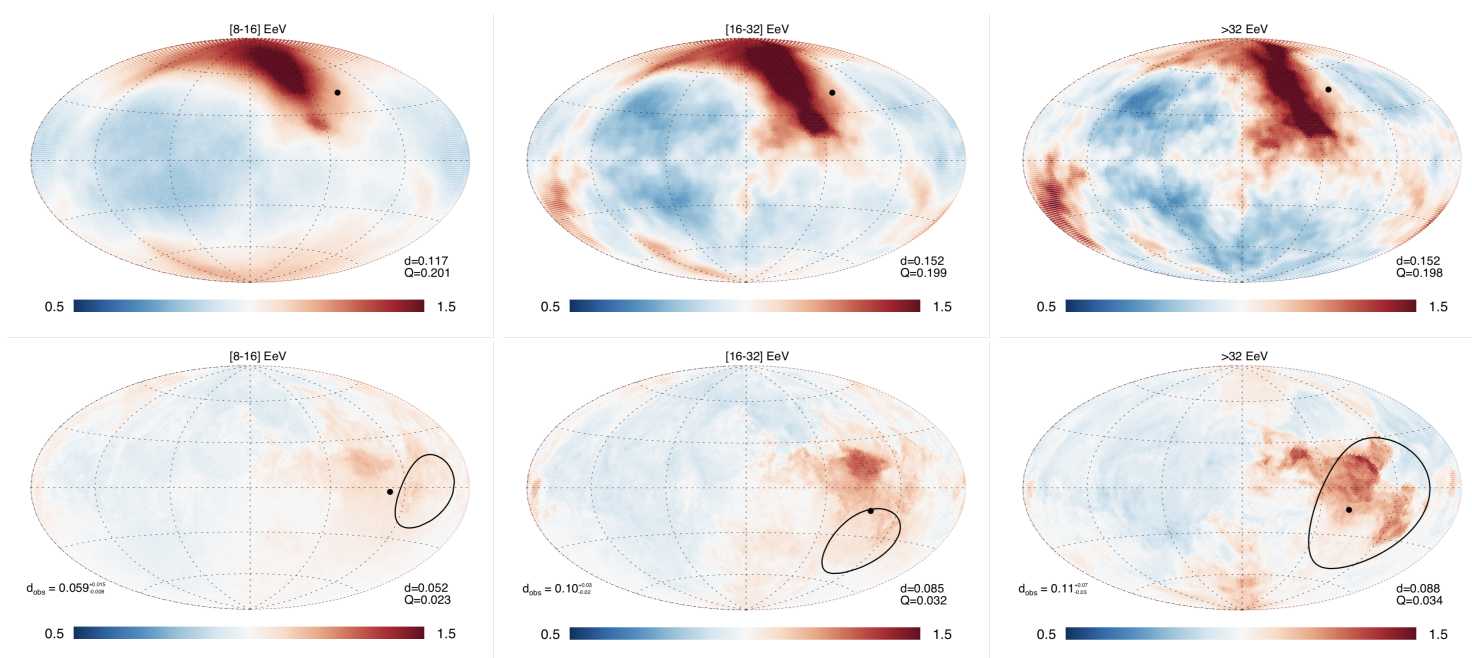

Figure 2: Top: illumination maps. Bottom: arrival maps. The energy bins are, from left to right: 8-16, 16-32, > 32 EeV. The amplitude of the model dipole and quadrupole moments are indicated on the right side legend of each map. The dipole values reported by Auger [2] are indicated on the left of the arrival maps. The closed curves show the Auger $68 \%$ CL dipole direction, and model predictions are shown with black dots.

\subsection{Model parameters and Chi-Square fitting}

The dipole components $d_{x}, d_{y}, d_{z}$ are the observables used in the fitting procedure. In equatorial coordinates, $d_{x}=d \cos \delta_{d} \cos \alpha_{d}, d_{y}=d \cos \delta_{d} \sin \alpha_{d}, d_{z}=d \sin \delta_{d}$, where $\alpha_{d}$ and $\delta_{d}$ are the right ascension and declination of the dipole. In terms of measurement, Auger applied Rayleigh analysis [2] on cosmic ray data set to reconstruct the dipole components via:

$$
d_{x} \simeq \frac{a_{1}^{\alpha}}{\langle\cos \delta\rangle} \pm \frac{\sqrt{2 / \mathscr{N}}}{\langle\cos \delta\rangle} ; d_{y} \simeq \frac{b_{1}^{\alpha}}{\langle\cos \delta\rangle} \pm \frac{\sqrt{2 / \mathscr{N}}}{\langle\cos \delta\rangle} ; d_{z} \simeq \frac{b_{1}^{\phi}}{\cos \ell_{\mathrm{obs}}\langle\sin \theta\rangle} \pm \frac{\sqrt{2 / \mathscr{N}}}{\cos \ell_{\mathrm{obs}}\langle\sin \theta\rangle}
$$

The probability density function $P$ of dipole $\left(d_{x}, d_{y}, d_{z}\right)$ is a 3D Gaussian function. Maximizing the likelihood $\mathscr{L}=P\left(\left(d_{x, o}, d_{y, o}, d_{y, o}\right) \mid\left(d_{x, c}, d_{y, c}, d_{y, c}\right)\right)$ is the same as minimizing the chi-square per

\footnotetext{
${ }^{2}$ The rigidity of cosmic ray, defined as energy divided by charge, is binned so that the bin centers are $\log _{10}[R(V)]=$ $17.4,17.5,17.6,17.8,17.9,18.0,18.1,18.2,18.3,18.4,18.5,18.6,18.7,18.75,18.8,18.9,19.0,19.2,19.4,19.5,19.6$, 19.8 and 20.0 plus a bin $\log _{10}[R(V)]<17.35$.
} 
observable $\chi^{2}=\left[\sum_{i=1}^{n}\left(C_{i}-O_{i}\right)^{2} / \sigma_{i}^{2}\right] / n$, where $O$ is observation with variance $\sigma^{2}, C$ is the result from our model, $n$ is the total number of observables ${ }^{3}$. For example, if $>8 \mathrm{EeV}$ is treated as one single bin, then $n=3$. If $>8 \mathrm{EeV}$ is treated as three bins $(8-16,16-32,>32) \mathrm{EeV}$ then $n=9$.

\subsection{Treatment of exposure}

Given that the Pierre Auger Observatory has only partial exposure, it is important to predict what dipole anisotropy Auger would report for a given model. The full-sky map can be expressed in terms of spherical harmonics $Y_{\ell m}$ and its coefficient $a_{\ell m}$ [16]:

$$
I(\mathbf{n})=\sum_{\ell \geq 0} \sum_{m=-\ell}^{\ell} a_{\ell m} Y_{\ell m}(\mathbf{n}) \quad a_{\ell m}=\int I(\mathbf{n}) Y_{\ell m}(\mathbf{n}) \mathrm{d} \Omega .
$$

With partial coverage, the observatory would detect $\mu(\mathbf{n}) I(\mathbf{n})$, with $\mu(\mathbf{n})$ being the directional exposure function. One can calculate the pseudo-coefficients:

$$
\tilde{a}_{\ell m}=\int \mu(\mathbf{n}) I(\mathbf{n}) Y_{\ell m}(\mathbf{n}) \mathrm{d} \Omega \equiv \sum_{\ell^{\prime} \geq 0} \sum_{m^{\prime}=-\ell}^{\ell} K_{[\ell m]\left[\ell^{\prime} m^{\prime}\right]} a_{\ell^{\prime} m^{\prime}} .
$$

Therefore, one can then reconstruct the coefficients $\bar{a}_{\ell m}$ from pseudo-coefficients $\tilde{a}_{\ell m}$ via $K^{-1}$ :

$$
\bar{a}_{\ell m}=\sum_{\ell^{\prime} \geq 0} \sum_{m^{\prime}=-\ell}^{\ell} K_{[\ell m]\left[\ell^{\prime} m^{\prime}\right]}^{-1} \tilde{a}_{\ell^{\prime} m^{\prime}}
$$

Using this method (denoted by 'K-inverse') we can calculate the anisotropy predicted from our model with consideration of partial exposure.

We have also applied the K-inverse method on the public Auger data [1] used by Auger in their Rayleigh analysis [1], to assess systematic errors and impact of partial sky coverage in the observation; see Table 1 for comparison. (With full-sky coverage and no observational systematics, the methods give identical results.) The differences between Rayleigh analysis and K-inverse methods are mainly manifested in $d_{z}$. This is expected because the directional exposure is zero for declination above 45 degree and it is difficult to measure $d_{z}$ with such incomplete coverage. For Auger, the Rayleigh analysis is preferred because it is expected to be less sensitive to the systematics of the experiment. The Auger data events list is only available for the 4-8 EeV and $>8 \mathrm{EeV}$ [1], so we cannot perform the K-inverse analysis for the 8-16, 16-32, > $32 \mathrm{EeV}$ bins individually. For these energy bins we just compare to the results given in [2] using the Rayleigh method.

\section{Results}

The impact of varying the EGMF field strength, fixing the EGMF and GMF coherence lengths to their best-fit values, is shown in Figs. 3 and 4 for the Auger composition and pure proton composition, respectively. As seen in Figure 3, the effect of the EGMF is to move the intrinsic dipole of source directions (which is in the CMB direction), via the magnetic horizon effect, so that the

\footnotetext{
${ }^{3}$ The diffusion coefficient $D_{\mathrm{EG}}\left(B_{\mathrm{EGMF}}, \lambda_{\mathrm{EGMF}}\right.$, rigidity), has the property that over the relevant rigidity domain, different combinations of $B_{\mathrm{EGMF}}$ and $\lambda_{\mathrm{EGMF}}$ produce a similar $D_{\mathrm{EG}}$ [15]. Thus the parameters $B_{\mathrm{EGMF}}$ and $\lambda_{\mathrm{EGMF}}$ are somewhat degenerate and there are effectively two degrees of freedom at our disposal to optimize the fit to the anisotropy, $D_{\mathrm{EG}}$ and $\lambda_{\mathrm{GMF}}$, with minor additional improvements possible within the freedom of composition model variations.
} 

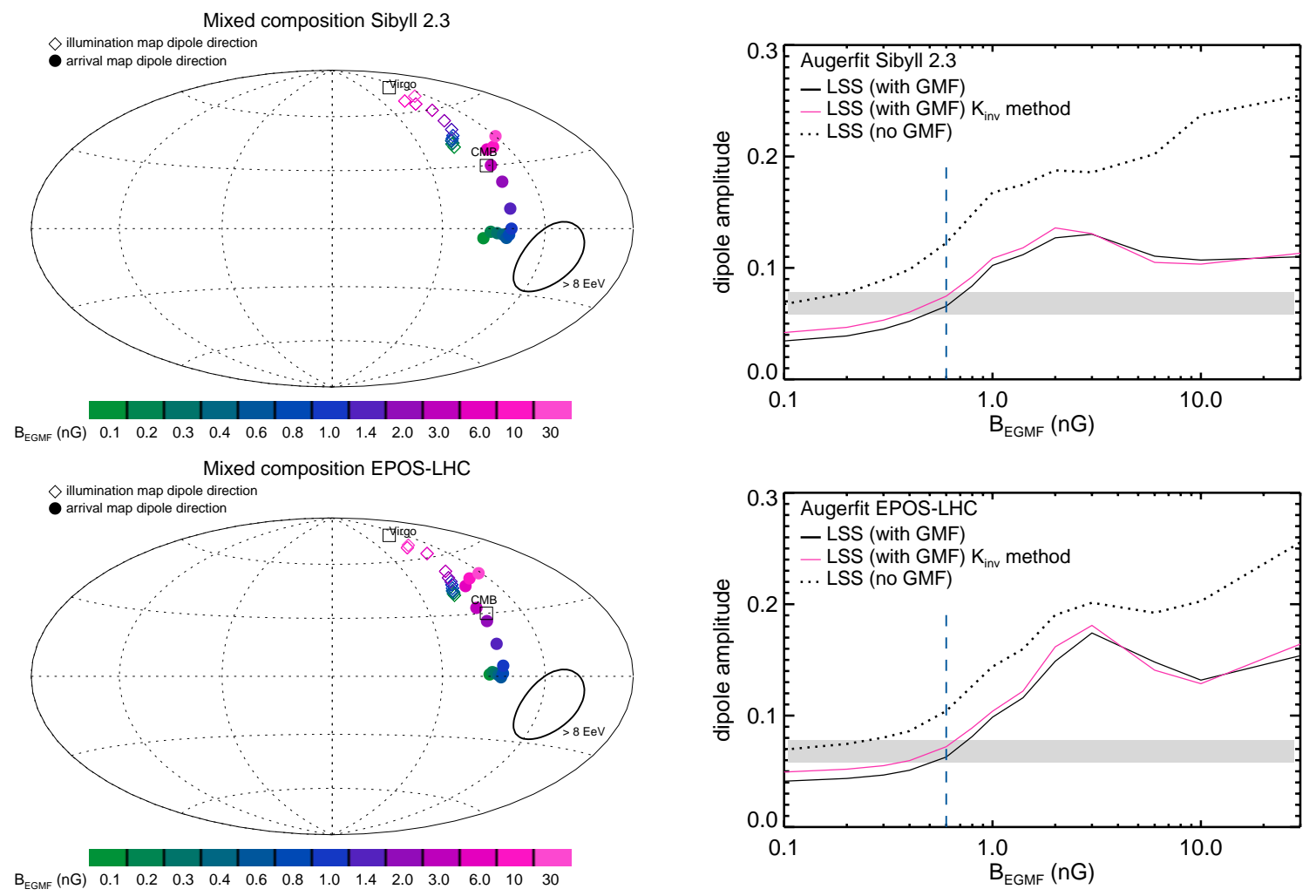

Figure 3: Effect of varying the EGMF field strength on the $>8 \mathrm{EeV}$ dipole direction (left) and amplitude (right), for mixed composition using Sibyll 2.3 (above) and EPOS-LHC (below). The GMF coherence length is fixed to $75 \mathrm{pc}$, its best fit value. The closed curves in the left panels show the $68 \%$ confidence level Auger dipole direction. The shaded region in the right panels shows the $68 \%$ confidence level band in Auger dipole amplitude.
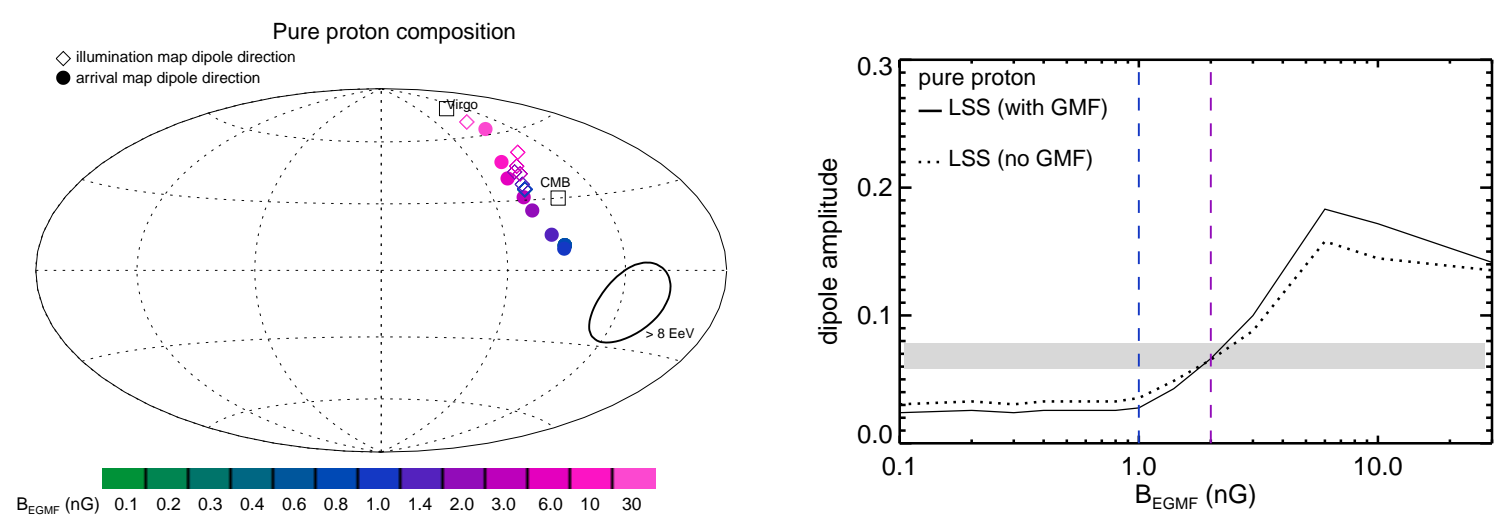

Figure 4: Same as Figure 3, but for pure proton model. Unlike the analysis for mixed composition, no choice of $B_{\mathrm{EGMF}}$ gives a good fit to both direction and magnitude. The purple vertical dashed line indicates the $B_{\mathrm{EGMF}}=2 \mathrm{nG}$ needed to fit the magnitude, but the direction (purple dot) is very far from the observed direction. The blue dot with $B_{\mathrm{EGMF}}=1$ nG gives the closest (but not good) fit to the direction, but the amplitude is far from observed amplitude.

illumination map dipole is in the general direction of the Virgo supercluster. The main effect of the GMF is to shift the IM dipole direction to the observed dipole direction and to reduce its amplitude to the observed value. The parameters giving the best overall fit are $B_{\mathrm{EGMF}} \sim 0.6 \mathrm{nG}, \lambda_{\mathrm{EGMF}} \sim 0.2$ 


\begin{tabular}{cccccc}
\hline $\begin{array}{c}\text { Energy } \\
(\text { EeV) }\end{array}$ & Observable & $\begin{array}{c}\text { Auger data set } \\
\text { Rayleigh analysis }\end{array}$ & $\begin{array}{c}\text { Auger data set } \\
\text { K-inverse method }\end{array}$ & $\begin{array}{c}\text { model } \\
\text { full-sky }\end{array}$ & $\begin{array}{c}\text { model } \\
\text { K-inverse }\end{array}$ \\
\hline \multirow{3}{*}{$>8$} & $d_{x}$ & $-0.011 \pm 0.010$ & $-0.009 \pm 0.010$ & -0.022 & -0.024 \\
& $d_{y}$ & $0.059 \pm 0.010$ & $0.057 \pm 0.010$ & 0.039 & 0.040 \\
& $d_{z}$ & $-0.026 \pm 0.015$ & $-0.039 \pm 0.013$ & -0.041 & -0.047 \\
\hline
\end{tabular}

Table 1: Comparison of the Auger dipole determined with Rayleigh and K-inverse methods, to our model predictions for full-sky dipole and for dipole with Auger exposure, calculated by the K-inverse method.

Mpc and $\lambda_{\mathrm{GMF}} \sim 75 \mathrm{pc}$. This result is insensitive to the hadronic models tested (EPOS-LHC and Sibyll 2.3).

As can be seen in the left panel of Fig. 4, it is impossible in the pure proton case to account for the dipole direction, for any choice of $B_{\mathrm{EGMF}}$, within the framework of our LSS model and our treatment of EGMF and GMF. This is due to the reduced impact of the GMF deflections for $Z=1$ [10]. For the value of $B_{\mathrm{EGMF}}$ giving the best fit to the amplitude, the predicted direction is vastly different than observed.

Our best-fit predictions for the dipole anisotropy above $8 \mathrm{EeV}$ are given in Table 1 . All numbers except 'model full-sky' are the dipole components if the arrival map is fitted with monopole and dipole (no higher multipoles). In the case of 'model full-sky', all multipole moments of the full-sky map of our model are calculated. In this case, the quadrupole amplitude is $Q=0.023$, which is compatible with experimental observation $Q=0.032 \pm 0.014$.

Figure 2 shows the illumination and arrival direction sky maps, for energy bins 8-16, 16-32 and $>32 \mathrm{EeV}$, using the overall best-fit parameters. The predicted dipole amplitudes for these 3 bins are $d_{8-16}=0.052, d_{16-32}=0.085, d_{>32}=0.088$, all of them are compatible with observations. The evolution of the dipole amplitude with energy is well described. The quadrupole amplitudes for these 3 bins are $Q_{8-16}=0.023, Q_{16-32}=0.032, Q_{>32}=0.034$.
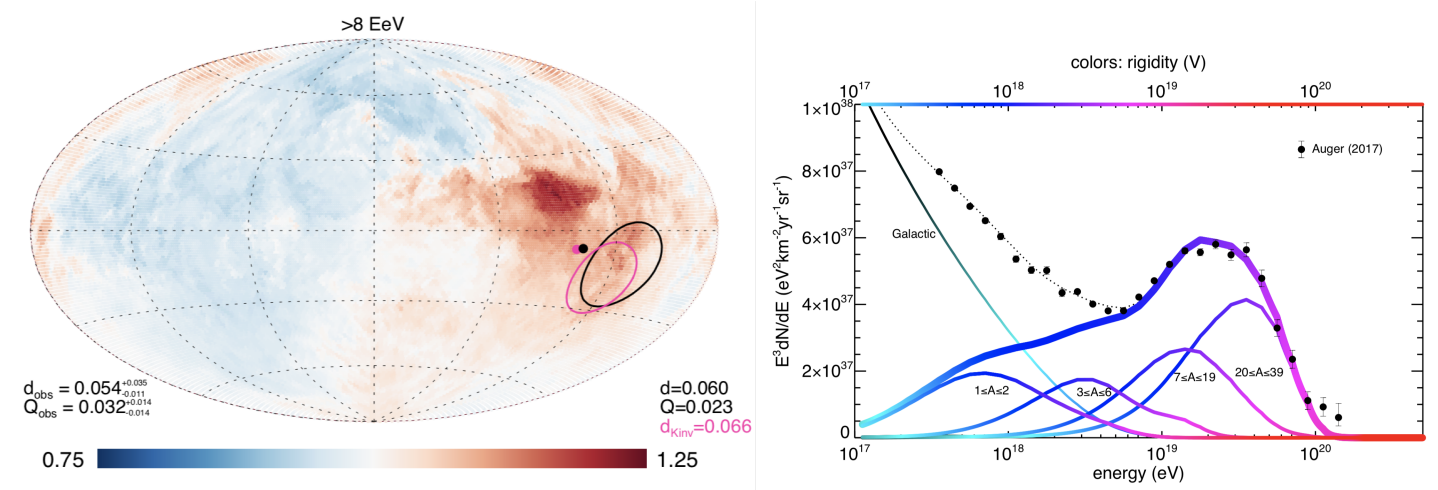

Figure 5: Left: Arrival direction map for UHECRs with $E>8 \mathrm{EeV}$; the predicted (observed) amplitude of the dipole and quadrupole moments are indicated in the right (left) legends. The $68 \% \mathrm{CL}$ dipole anisotropy direction from the Auger Rayleigh analysis and our K-inverse analysis are in black and pink. The all-sky and as-observed (partial-sky) predicted dipole direction from our model are the black and pink dots. Right: Spectrum and composition of the UHECRs used from MUF19, with mean rigidities indicated by color. 


\section{Conclusions}

Assuming the distribution of UHECR sources follows the local large scale distribution of matter and using a UHECR spectrum and composition fit to the Auger data, and taking into account diffusion in extragalactic magnetic field and deflections in the Galactic magnetic field, we obtain excellent agreement with the anisotropy of extragalatic cosmic rays $(E>8 \mathrm{EeV})$ reported by Auger (Fig. 5). The energy dependence is also well-described (Fig. 2). The best-fitting values for the EGMF field strength and EGMF and GMF coherence lengths are $B_{\mathrm{EGMF}} \sim 0.6 \mathrm{nG}, \lambda_{\mathrm{EGMF}} \sim 0.2$ $\mathrm{Mpc}$ and $\lambda_{\mathrm{GMF}} \sim 75 \mathrm{pc}$ (see Fig. 3). This result does not depend on the hadronic model used (EPOS-LHC or Sibyll 2.3) nor on the reconstruction method used to take into account the Auger exposure (Rayleigh or K-inverse). If the composition above $8 \mathrm{EeV}$ were pure proton, the impact of the GMF on modifying the extragalactic illumination would be lessened and it would not be possible to fit simultaneously the dipole amplitude and direction, with the direction being particularly problematic (see Fig. 4).

We thank Marco Muzio for the MUF19 fits, Yehuda Hoffman for his permission to use the density field from [11] and Olivier Deligny for valuable discussions; GRF acknowledges the help of colleagues in the Pierre Auger collaboration. The research of NG was supported by New York University and the Simons Foundation, and that of GRF by NSF-1517319.

\section{References}

[1] Pierre Auger Collaboration, Aab, A. et al. 2017, Science, 357, 1266

[2] Pierre Auger Collaboration, Aab, A. et al, Large-scale Cosmic-Ray Anisotropies above 4 EeV Measured by the Pierre Auger Observatory 868 (2018) 4. doi:10.3847/1538-4357/aac689.

[3] Farrar, G. R., \& Piran, T. 2000, Physical Review Letters, 84, 3527

[4] Silvia Mollerach and Esteban Roulet, Phys. Rev. D 99, 103010 (2019)

[5] S. Hackstein, F. Vazza, M. BrÃijggen, J. G. Sorce, and S. Gottlãúuber, Mon. Not. R. Astron. Soc. 475, 2519 (2018).

[6] N. Globus, T. Piran, Y. Hoffman, E. Carlesi, and D. Pomare`de, Mon. Not. R. Astron. Soc. 484, 4167 (2019).

[7] Globus, N., \& Piran, T. 2017, ApJL, 850, L25

[8] Jansson, R. \& Farrar, G. R., 2012, ApJ, 757, 14

[9] Parizot, E. 2004, Nuclear Physics B Proceedings Supplements, 136, 169

[10] Farrar, Glennys R. and Sutherland, Michael S. JCAP05(2019)004

[11] Hoffman, Y. and Carlesi, E. and Pomarède, D. and Tully, R. B. and Courtois, H. M. and Gottlöber, S. and Libeskind, N. I. and Sorce, J. G. and Yepes, G., Nature Astronomy, vol 2, p 680-687 (2018)

[12] Muzio, M. S., Unger, M., and Farrar, G. R., Progress towards characterizing ultrahigh energy cosmic ray sources, arXiv:1906.06233

[13] D. Veberic [Pierre Auger Collaboration], arXiv:1708.06592 [astro-ph.HE].

[14] Tully R. B., Courtois H., Hoffman Y., Pomarède D., 2014, Nature, 513, 71

[15] Globus, N., Allard, D., Parizot, E., 2008, A\&A, 479, 97

[16] Deligny, O., Astroparticle Physics Volume 104, January 2019, Pages 13-41 